\title{
Buyang huanwu decoction promotes angiogenesis via vascular endothelial growth factor receptor-2 activation through the PI3K/Akt pathway in a mouse model of intracerebral hemorrhage
}

Han-Jin Cui ${ }^{1,3}$, A-Li Yang ${ }^{2,3}$, Hua-Jun Zhou ${ }^{3,4}$, Cong Wang ${ }^{1,3}$, Jie-Kun Luo ${ }^{1,3}$, Yuan Lin³ ${ }^{3}$ Yan-Xia Zong ${ }^{1,3}$ and Tao Tang ${ }^{1,3^{*}}$

\begin{abstract}
Background: Intracerebral hemorrhage $(\mathrm{ICH})$ is a fatal subtype of stroke that lacks effective treatments. Angiogenesis following ICH is an important response mediating brain recovery and repair. Phosphorylation of vascular endothelial growth factor receptor 2 (pVEGFR2) via PI3K/Akt signaling plays a key role in mediating cellular processes involved in repair, such as mitogenesis, angiogenesis, and vascular permeability. This study aimed to investigate the potential effects of Buyang Huanwu Decoction (BYHWD), a traditional Chinese medicine formula, on angiogenesis by VEGFR2 activation through the phosphatidylinositol 3 kinase (PI3K)/Akt signaling pathway in a mouse model of ICH.
\end{abstract}

Methods: Adult male Kunming mice $(n=50)$ were randomly assigned into sham and ICH-operated groups and treated with one of the followings SU5416 (VEGFR2 inhibitor), BYHWT and BYHWT + SU5416. ICH was induced in mice by injecting collagenase (type VII) into the right globus pallidus of the mouse brain. BYHWD $(4.36 \mathrm{~g} / \mathrm{kg}) \mathrm{was}$ administrated in mice by intragastric infusion. Neurological function was evaluated in mice by a modified Neurological Severity Scores (mNSS) as well as corner turn and foot-fault tests. Angiogenesis was examined by intraperitoneal injection of 5-bromodeoxyuridine (BrdU) in mice to quantify new brain vessel growth. SU5416 treatment and assessment of VEGFR2 phosphorylation as well as alterations in PI3K/Akt signaling were performed to determine whether the effect of BYHWD on angiogenesis was partly mediated by phosphorylation of VEGFR2 via the PI3K/Akt signaling pathway.

Results: We show that BYHWD treated mice exhibited (i) significantly better recovery from neurological dysfunction, (ii) increased $\mathrm{BrdU}^{+}$nuclei in $\mathrm{VWF}^{+}$dilated brain vessels and (iii) higher VEGFR2 phosphorylation immunoreactivity in brain microvessels $(P<0.05)$, (iv) higher expression of PI3K and pAkt at the protein level $(P<0.05)$ when compared to untreated ICH mice. These beneficial effects were reversed by SU5416 $(P<0.05)$.

Conclusions: BYHWD promoted neurological recovery and angiogenesis after ICH in mice by enhancing VEGFR2 phosphorylation through the PI3K/Akt signaling pathway.

Keywords: Intracerebral hemorrhage, Angiogenesis, Vascular endothelial growth factor receptor-2, Phosphorylation, PI3KJAkt, Buyang huanwu decoction

\footnotetext{
* Correspondence: falcontang@126.com

'Institute of integrative medicine, Xiangya Hospital, Central South University, Changsha 410008, Hunan, China

${ }^{3}$ Key Lab of Chinese Gan of SATCM, Changsha 410008, Hunan, China

Full list of author information is available at the end of the article
} 


\section{Background}

Spontaneous intracerebral hemorrhage $(\mathrm{ICH})$ is one of the most lethal types of stroke [1] with the highest mortality and morbidity [2]. Conventional therapies for $\mathrm{ICH}$ consist of hematoma removal, edema attenuation and intracranial pressure reduction. However, the effectiveness of $\mathrm{ICH}$ therapy is far from satisfactory [3]. It is therefore important and necessary to explore new strategies for ICH treatment.

Recent studies on pathophysiological mechanisms of ICH impairment mainly focus on secondary brain injury and neuronal death caused by inflammation iron accumulation. Inflammation is mediated by inflammatory cells (blood-derived leukocytes, macrophages, microglia, astrocytes, mast cells) activation and their local release of cytokines, proteases, prostaglandins, proteases and so on $[4,5]$. Excessive iron accumulation after $\mathrm{ICH}$ due to heme degradation can stimulate reactive oxygen species (ROS) formation causing neurotoxicity and neurodegeneration [6]. Despite these impairment factors, there are also endogenous mechanisms of brain self-repair after stroke, which include angiogenesis.

Normal brain function requires unrestricted blood flow to supply glucose and oxygen to neurons. Following a damaging $\mathrm{ICH}$ event, new microvasculature formation is required for brain recovery by promoting oxygen and metabolite exchange and removing necrotic debris $[7,8]$. Angiogenesis refers to the establishment of new vessels from pre-existing vasculature by such processes as sprouting, pruning and intussusception. It starts with stimulation of endothelial cells (ECs) that line the luminal surface of blood vessels, and occurs under both physiological and pathological conditions $[9,10]$. During angiogenesis, a highly regulated process, the most potent pro-angiogenic signaling cascades are initiated by vascular endothelial growth factor (VEGF) binding to EC-resident VEGF receptors (VEGFR) like VEGFR1, VEGFR2 and VEGFR3 [9]. Of these, VEGFR2 transduces angiogenic signals after binding VEGF, while VEGFR1 transduces only weak signals for ECs growth and survival, and VEGFR3 is generally restricted to lymphatic endothelia [11]. VEGF expression has been evaluated and shown to be an important component of the response after brain injury [12]. We have previously demonstrated that angiogenesis can occur following $\mathrm{ICH}$ in brains, and are able to specifically detect VEGF and VEGFR2 around the hematoma [13].

VEGF binding to VEGFR2 leads to the receptor dimerization, protein kinase activation, autophosphorylation, and finally, the initiation of several signaling cascades [14]. Amongst the endothelial cell signaling pathways, PI3K/Akt is known to be a major regulator of proliferation, migration and survival of ECs as well as vascular permeability. VEGFR2 activation is believed to be the "switch" that activates the downstream PI3K/Akt signaling cascade
[15]. SU5416, a potent selective inhibitor of VEGFR2 tyrosine kinase catalysis, 3-[2, 4- dimethylpyrrol-5-yl methylidenyl] -2-indolinone, has been shown to reduce tumor angiogenesis and growth in animal models [16-18].

Traditional Chinese medicine (TCM) has been shown to have positive effects in stroke [19]. As a classical TCM prescription for stroke, Buyang Huanwu Decoction (BYHWD) shows substantial neuroprotective, pro-angiogenic and function-improving effects in animal models of focal cerebral ischemia [20-22], while also possessing desirable clinical efficacy for both hemorrhagic and ischemic stroke [23]. Furthermore, we have shown previously that BYHWD could upregulate VEGF and VEGFR2 after ICH [24]. Since VEGFR2 activation can efficiently stimulate angiogenesis [25], we hypothesized that BYHWD could likewise promote angiogenesis in the ICH model through VEGFR2 stimulation. Therefore, we were interested in studying the effects of BYHWD on the recovery of the brain after hemorrhagic injury as well as VEGFR2 phosphorylation and its signaling pathway mechanisms.

\section{Methods}

\section{Animal preparation}

Studies were carried out on adult male Kunming mice (25-30 g, 8-10 weeks of age) obtained from the Experimental Animal Science Center of Central South University (CSU). All animals were housed under identical conditions (room temperature at $25^{\circ} \mathrm{C}, 12 \mathrm{~h}$ light-dark cycle) and allowed free access to food and water. The experimental protocol was performed in compliance with guidelines of CSU and the NIH Guide for the Care and Use of Laboratory Animals (NIH Publications No. 80-23) and was approved by the Institutional Animal Care and Use Committee of CSU (2010-xy-0106).

\section{BYHWD preparation}

According to the original prescription from the "TCM Prescriptions Dictionary", BYHWD consists of seven Chinese herbs (Table 1). All dried crude herbs were purchased from the Chinese Medicinal Pharmacy of Xiangya Hospital, Changsha, China. Voucher specimens were prepared and deposited at the Laboratory of Institute of Integrative Medicine, Central South University. To maintain the consistency of the herbal chemical ingredients, all components were obtained from the original sources and extracted according to standards listed in the National Pharmacopoeia of China. The herbs were cut, mixed, and decocted by boiling in distilled water at $100^{\circ} \mathrm{C}$ for $30 \mathrm{~min}$. The solution was then freeze-dried under vacuum, and ground into powder (yield: 14.3\%). The powder was dissolved in distilled water to a final concentration of $0.132 \mathrm{~g} / \mathrm{ml}$. 
Table 1 Components of the buyang huanwu decoction

\begin{tabular}{|c|c|c|c|c|c|}
\hline Latin name & Family & English name & Chinese name & Part used & Batch number \\
\hline Radix astragali & Leguminosae & Astragalusmongholicus & Huang qi & Root & 091201 \\
\hline Radix paeoniaeRubra & Ranunculaceae & Red peony root & Chi shao & Root & 091214 \\
\hline Radix angelicaeSinensis & Umbelliferae & Angelica root & Dang gui & Root & 091206 \\
\hline RhizomaLigusticiChuanxiong & Umbelliferae & Sichuan lovage rhizome & Chuanxiong & Root & 100304 \\
\hline FlosCarthami & Feverfew & Carthamustinctorius & Hong hua & Flower & 100329 \\
\hline Semen persicae & Rosaceae & Peach seed & Tao ren & Seed & 091218 \\
\hline PheretimaAspergillum & Megascolecidae & Earthworm (Lumbricus) & Di long & Whole animal & 091222 \\
\hline
\end{tabular}

In the order listed above, the herbs were combined in a 60:6:4.5:3:3:3 ratio (dry weight).

\section{$\mathrm{ICH}$ induction}

After fasting overnight, the mice were anesthetized with $5 \%$ chloral hydrate $(300 \mathrm{mg} / \mathrm{kg}$ IP) and then fixed on a stereotaxic frame (Stoelting Co., Chicago, IL, USA) in the prone position. Following a scalp incision and drilling of a small cranial bur hole, $0.075 \mathrm{U}$ of collagenase (type VII) in $0.5 \mu \mathrm{l} 0.9 \%$ sterile saline was slowly injected into the right globus pallidus $(0.5 \mathrm{~mm}$ posterior, $2.0 \mathrm{~mm}$ lateral to bregma and $4.0 \mathrm{~mm}$ ventral to cortical surface) of the mouse brain, using a $1-\mu \mathrm{l}$ Hamilton syringe. The infusion was administered over $2 \mathrm{~min}$, with the needle kept in place for an additional 5 minutes thereafter. In the sham-operated group, $0.5 \mu \mathrm{l} 0.9 \%$ sterile saline without collagenase was injected into the same site.

\section{Experimental protocol}

Mice were randomly assigned to both sham and ICH operated groups and treated with one of the followings: SU5416, BYHWT and BYHWT + SU5416 $(n=10$, per group). Twenty-four hours after ICH induction, 5bromodeoxyuridine (BrdU, Roche, Indianapolis, IN, USA) was given $(50 \mathrm{mg} / \mathrm{kg} / \mathrm{d})$ by intraperitoneal injection. The SU5416-treated and BYHWT + SU5416-treated groups were administrated with SU5416 (10 mg/kg, Santa Cruz, Dallas, Texas, USA) in distilled water (DW) containing 1\% dimethyl sulfoxide (DMSO, Calbiochem, La Jolla, CA, USA) by intraperitoneal injection. Control groups received 1\% DMSO in their respective cocktails. BYHWD $(4.36 \mathrm{~g} / \mathrm{kg})$ was given to BYHWD-treated and BYHWT + SU5416-treated groups intragastrically. Equal volume of DW was given to all other groups. The dose of BYHWD of $4.36 \mathrm{~g} / \mathrm{kg}$ was chosen from the preliminary tests for the recovery of motor function in ICH animals (data not shown). Administration of drug treatments was performed $24 \mathrm{~h}$ after ICH induction, once a day for 7 days.

\section{Behavioral tests}

Behavioral tests were carried out at day 1, 3, 7 after ICH. Two observers, blinded to the experimental design, scored the animals independently and the scores were averaged. The tests were composed of a modified
Neurological Severity Score (mNSS) [26], corner turn test [27] and foot-fault test [28].

1) Neurological score: An eighteen point neurological score was employed: (normal score, 0; maximal deficit score, 18). For severity scores of injury, 1 score point is awarded for the inability to perform the test or for the lack of a tested reflex; thus, the higher score, the more severe the injury [26].

2) Corner turn test: Mice were allowed to proceed into a corner, the angle of which was 30 degrees. To exit the corner, individual mice could turn either left or right, and the direction taken was then recorded. This was repeated 10 to 15 times per animal, with at least 30 seconds between trials, and the percentage of right turns was calculated [27].

3) Foot-fault test: Mice were tested for placement dysfunction of forelimbs with the modified foot-fault test. Animals were set on an elevated grid surface $\left(85.8 \times 2.5 \mathrm{~cm}^{2}\right.$, with grids of different sizes) and placed their paws on the wire while moving along the grid. With each weight-bearing step, the paw may fall or slip between the wire. Each such event was recorded as a foot fault. The total number of steps (movement of each forelimb) that the mice used to cross the grid was counted, and the total numbers of foot faults for the left forelimb were recorded. Data are presented as the percentage of foot faults per the total number of steps [28].

\section{Specimen preparation}

Randomly chosen animals from each group were deeply anesthetized with chloral hydrate $(300 \mathrm{mg} / \mathrm{kg})$. For immunohistochemistry, animals were transcardially perfused with $0.9 \%$ saline followed by $250 \mathrm{ml}$ ice-cold $4 \%$ paraformaldehyde in $0.1 \mathrm{M}$ phosphate buffer ( $\mathrm{PB}$, $\mathrm{pH}$ 7.4). The excised brains were post-fixed in the same fixative for $2 \mathrm{~h}$, then transferred to $20 \%$ and then $30 \%$ sucrose in $0.1 \mathrm{M} \mathrm{PB}\left(\mathrm{pH}\right.$ 7.4) sequentially at $4^{\circ} \mathrm{C}$ until sinking. Brains were cut into $30 \mu \mathrm{m}$ coronal sections at $-20^{\circ} \mathrm{C}$ with a cryostat (CM1900, Leica, Germany), some 
of which were collected in $0.01 \mathrm{M}$ phosphate-buffered saline $(\mathrm{pH} 7.4)$ and stored at $4^{\circ} \mathrm{C}$.

For Western blot analysis, mice were transcardially perfused with ice-cold $0.9 \%$ saline and then sacrificed by decapitation. The brains were immediately removed, and the tissues in the striatum, adjacent to the hematoma without the needle track, were dissected and stored in liquid nitrogen tank.

\section{Immunohistochemical analysis}

To detect proliferating cerebral microvascular ECs, immunohistochemical double-staining was performed. Sections were pretreated to denature DNA as follows: after immersion in $50 \%$ formamide $/ 2 \times$ saline-sodium citrate buffer (SSC, pH 7) at $65^{\circ} \mathrm{C}$ for $2 \mathrm{~h}$, sections were washed in $2 \times \mathrm{SSC}$ for $10 \mathrm{~min}$ then incubated in $2 \mathrm{~N} \mathrm{HCl}$ at $37^{\circ} \mathrm{C}$ for $30 \mathrm{~min}$. After nonspecific antigen blocking in 5\% bovine serum albumin (BSA, Sigma-Aldrich, St. Louis, MO, USA), sections were incubated with a mouse antiBrdU (1:200, Neomarker, Fremont, CA, USA) at $4^{\circ} \mathrm{C}$ overnight, then with biotinylated anti-mouse IgG (1:200, Vector Laboratories, Burlingame, CA, USA) for $1 \mathrm{~h}$ at $37^{\circ} \mathrm{C}$, followed by avidin-biotin-peroxidase complex (ABC) (1:100, Vector) for $1 \mathrm{~h}$ at $37^{\circ} \mathrm{C}$. Immunoreactivity was visualized with diaminobenzidine (DAB, Boster Biotech, Wuhan China). For labeling cerebral EC's, we used a rabbit anti-von Willebrand factor (vWF, 1:400, Chemicon International, Temecula, CA, USA) primary antibody in concert with ammonium nickel sulfate to enhance visualization. $\mathrm{BrdU}^{+} / \mathrm{vWF}^{+}$nuclei close to the hematoma were counted in four $250 \times 250 \mu \mathrm{m}$ areas in five sections through the stroke for each animal at $\times 40$ objective magnification using image J analysis software, by a researcher blind to the experimental design as previously described. Data were presented as the number of nuclei per $\mathrm{mm}^{2}$ $\left(\mathrm{N} / \mathrm{mm}^{2}\right)$.

For expression of phospho (p)-VEGFR2, sections were brought to room temperature and incubated in $3 \% \mathrm{H}_{2} \mathrm{O}_{2}$ for $15 \mathrm{~min}$. After washing sections 3 times in phosphatebuffered saline for 5 min each, non-specific binding was blocked in $5 \%$ bovine serum albumin (Sigma Aldrich) for $1 \mathrm{~h}$ at $37^{\circ} \mathrm{C}$. Sections were then incubated overnight at $4^{\circ} \mathrm{C}$ with a pVEGFR2 rabbit mAb (diluted 1:100, Cell Signaling Technology, Boston, MA, USA), with a biotinylated anti-rabbit immunoglobulin G (1:200) for $2 \mathrm{~h}$, and then with avidin-biotin-peroxidase complex (1:100, Vector) for $1 \mathrm{~h}$ at $37^{\circ} \mathrm{C}$. Immunoreactivity was visualized with diaminobenzidine (DAB, Boster Biotech). Areas of immunoreactivity were measured using Qwin software to determine gray scale.

To determine whether pVEGFR2 was expressed in ECs, immunofluorescence double labeling was performed. Tissue sections were first incubated at $4^{\circ} \mathrm{C}$ for $48 \mathrm{~h}$ with a mixture of 2 primary antibodies: mouse anti-vWF (1:200,
Chemicon Int) and either of the rabbit anti-phosphoVEGFR2 (1:100). The following secondary antibodies were then used: fluorescein isothiocyanate-conjugated goat anti-mouse antibody (1:50, Santa Cruz Biotech, Santa Cruz, CA, USA) for vWF and rhodamine-conjugated donkey anti-rabbit antibody (1:50, Santa Cruz) for pVEGFR2 detection. For a negative control, $1 \%$ bovine serum albumin was used instead of the primary antibody. These sections were scanned using a laser confocal microscope (LSM-510, Zeiss).

\section{Western blot assay}

Tissues from the right globus pallidus were homogenized in RIPA buffer containing $50 \mathrm{mM}$ Tris- $\mathrm{HCl}$ (pH 7.4), $150 \mathrm{mM} \mathrm{NaCl}, 1 \%$ sodium deoxycholate, $1 \%$ NP-40, $0.1 \%$ lauryl sodium sulfate, $1 \mathrm{mM}$ phenylmethylsulfonyl fluoride (PMSF), and 1\% inhibitor cocktail (BioBasic Inc, Markham, ON, Canada), and the homogenate was centrifuged at $14,000 \mathrm{~g}$ for $30 \mathrm{~min}$. Proteins $(50 \mu \mathrm{g})$ from the supernatant of each sample were then separated by SDS-PAGE and transferred onto polyvinylidene difluoride membranes, which were subsequently blocked in TBST buffer containing $20 \mathrm{mMTris}-\mathrm{HCl}, 5 \%$ skim milk, $150 \mathrm{mM} \mathrm{NaCl}$, and $0.05 \%$ Tween 20 (pH 7.5) for $2 \mathrm{~h}$ at room temperature. Membranes were incubated with either goat anti-p-VEGFR2 (1:200, Santa Cruz Biotech), rabbit-anti-VEGFR2 (1:1000, Abcam, Cambridge, UK), rabbit anti-PI3K (1:2000, Millipore-Merck, Darmstadt, GER), rabbit anti-Akt (1:2000, Cell Signaling Tech), rabbit anti-p-Akt (1:2000, Cell Signaling Tech) or mouse antiactin $\left(1: 5000\right.$, Abcam) antibodies for overnight at $4^{\circ} \mathrm{C}$. Subsequently, membranes were incubated with horseradish peroxidase-conjugated anti-goat IgG (1:5000, Promega, Madison, WI, USA), anti-rabbit IgG antibody (1:5000, Promega) or anti-mouse IgG (1:5000, Promega) antibodies for $2 \mathrm{~h}$ at room temperature. The immunopositive bands were visualized by chemiluminescence detection using SuperSignalWest Pico Chemilumescent Substrate (Thermo) and a Bio-Rad ChemiDoc XRS digital documentation system (Bio-Rad, Hercules, CA, USA). The optical density (OD) of each band was normalized against that of $\beta$-actin.

\section{Statistical analysis}

All data in this study are presented as mean \pm standard deviation (SD). Data were analyzed with Student's t test and one-way analysis of variance (ANOVA), followed by Scheffe's post hoc test. Differences were considered significant at $P<0.05$.

\section{Results}

Effect of BYHWD on the neurological outcomes of ICH mice On the first day, all four groups subjected to collagenase-induced ICH showed similar neurological deficits, while the sham-operated animals were almost 
free of neurological impairments. Evaluation of mNSS at later time points revealed that the neurological status of both the ICH group and BYHWD-treated group improved over time (a decrease in the mNSS) with the former significantly higher than the latter (more than 2 points higher at day 3 and $7, P<0.05)$. However, the scores were significantly $(P<0.05)$ higher in the SU5416-treated and BYHWD + SU5416-treated group compared with those in the ICH group (about 3 points higher at day 3 and 7) and BYHWD-treated group (more than 4 points higher at day 3 and 7 ), respectively (Figure $1 \mathrm{~A}, P<0.05, P<0.01$ ).

At day 3, the ICH group (about 20\%) and BYHWDtreated group (about 30\%) performed significantly better in the corner turn test than their inhibitor-treated counterparts, respectively $(P<0.05, P<0.01)$. BYHWD-treated mice significantly outperformed ICH mice (about 20\% lower at day 3 and 7 , respectively) (Figure $1 \mathrm{~B}, P<0.05$ ). Similarly, BYHWD-treated mice significantly outperformed ICH mice in the foot-fault test (about 20\% lower at day 3 and 7, respectively). Treatment with SU5416 worsened the response of the foot-fault test in BYHWDtreated (about 40\% higher at day 3 and 7, respectively) and ICH mice (about 30\% higher at day 3 and 7 , respectively) (Figure $1 \mathrm{C}, P<0.01, P<0.05$ ).

\section{BYHWD promoted angiogenesis via upregulating PVEGFR2} The effects of BYHWD on pVEGFR2 expression and angiogenesis in the brain of mice at 7 days after $\mathrm{ICH}$ were further examined using the double-labeling immunohistochemistry (Figure 2A-M). The results showed that pVEGFR2 expression was primarily localized to the vWF-immunoreactive ECs (Figure 2A), while pVEGFR2positive microvessels and $\mathrm{BrdU}^{+}$nuclei in $\mathrm{vWF}^{+}$vessels were rarely observed in either hemisphere of the shamoperated animals (Figure 2B, H, G and M). In contrast, pVEGFR2-positive microvessels and BrdU-labeled vessels in the ICH group appeared to be dilated and most were present in the perihematomal tissue (Figure 2C, I, G and $M)$. We observed fewer vessels positive for pVEGFR2 and BrdU in the SU5416-treated animals (Figure 2D, J, G and $\mathrm{M})$. In comparison, significantly more pVEGFR2-
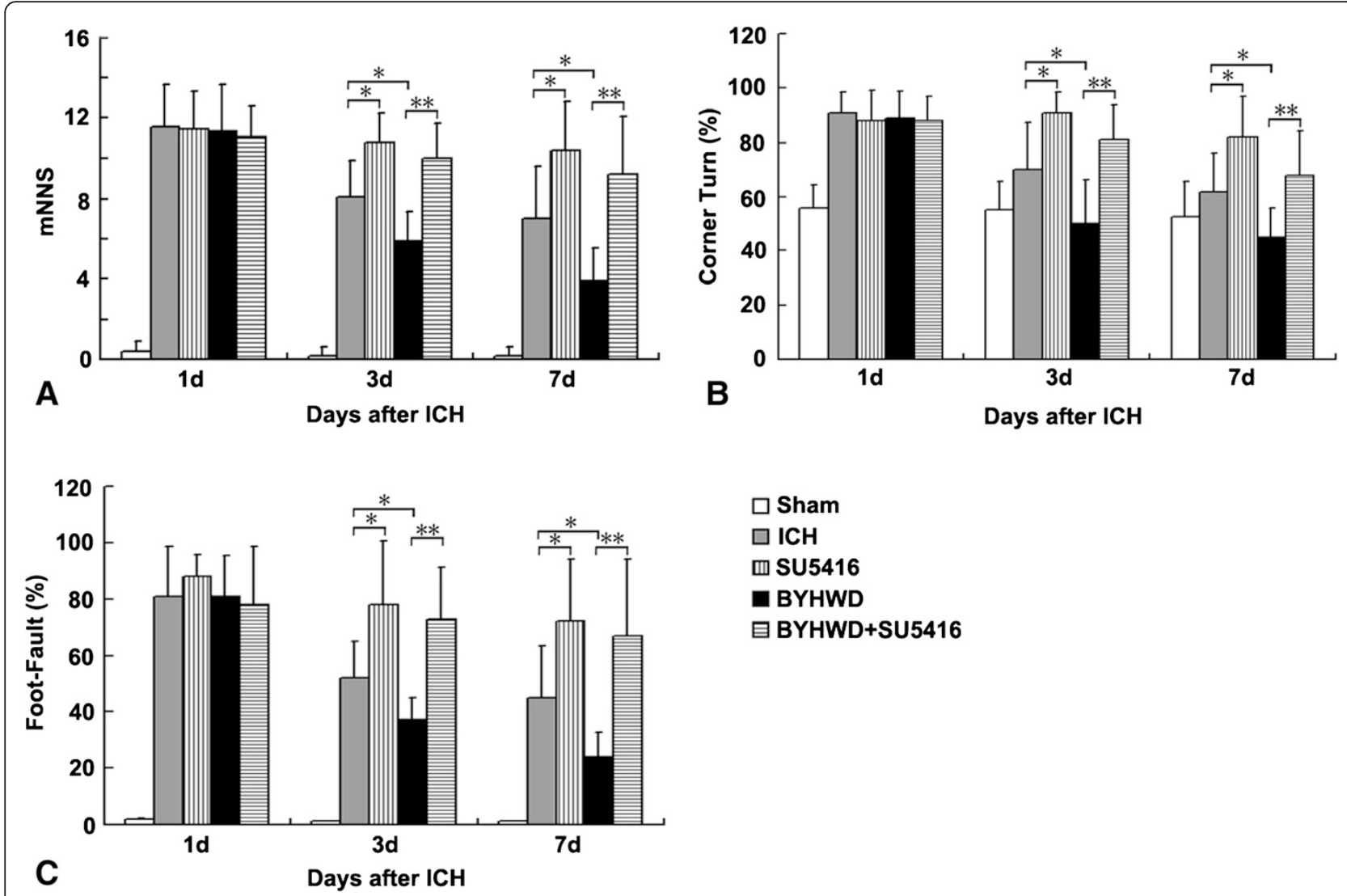

Figure 1 Effect of BYHWD on neurological outcomes in mice after ICH. Mice in the ICH group and BYHWD-treated group ( $3 \mathrm{~d}$ and $7 \mathrm{~d}$ ) showed a significantly lower mNSS (A) as well as better performance in corner turn (B) and foot-fault (C) tests than their inhibitor-treated counterparts. Similar differences were found between BYHWD-treated group and ICH group. The percentage of right turns (B) and foot faults (C) from each group was compared with the total number of steps. Data represent the mean $\pm S D, n=10 . *, P<0.05 ;{ }^{* *}, P<0.01$ ). Data were analyzed with Student's $t$ test and one-way analysis of variance (ANOVA), followed by Scheffe's post hoc test. 


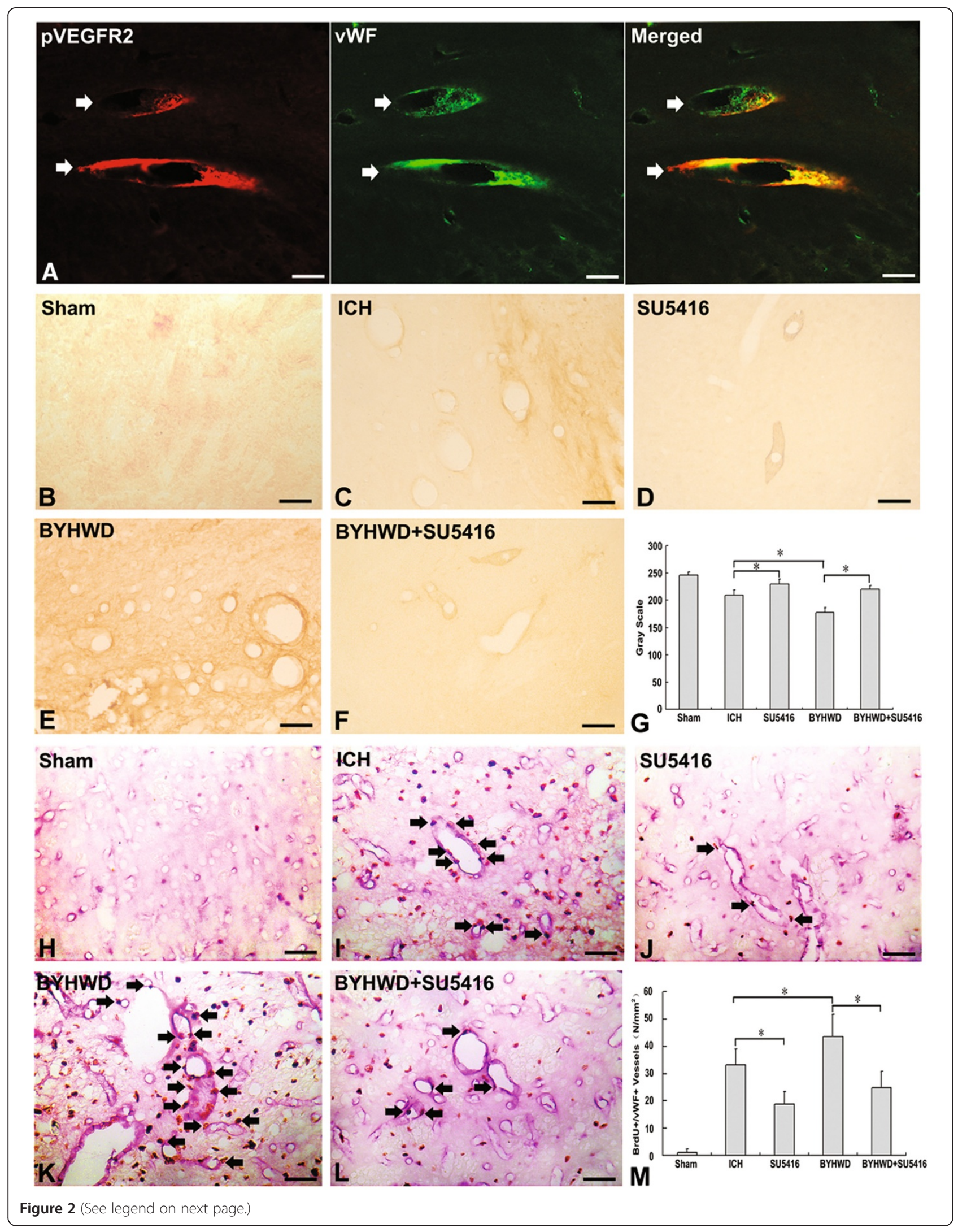


(See figure on previous page.)

Figure 2 BYHWD upregulates pVEGFR2 expression and angiogenesis following ICH. Double-labeling revealed that immunoreactivity for pVEGFR2 (red, arrow) overlapped with $\mathrm{VWF}^{+}$cells (green, arrow) (A). pVEGFR2-positive microvessels and BrdU ${ }^{+}$nuclei (brown, arrow) were shown in $\mathrm{VWF}^{+}$vessels (blue) in either hemisphere of the sham-operated animals (B and $\left.\mathbf{H}\right)$, ICH group (C and I), SU5416-treated (D and J), BYHWD- treated $\left(\mathbf{E}\right.$ and $\mathbf{K}$ ) and BYHWD + SU5416-treated ( $\mathbf{F}$ and $\mathbf{L}$ ) groups. The immunoreactivity of p-VEGFR2 (G) and the number of BrdU $\mathrm{UWWF}^{+}(\mathbf{M})$ nuclei were compared between BYHWD-treated and non-treated groups.

positive microvessels and BrdU-labeled vessels could be found in the vicinity of the lesion in the BYHWD treatment groups (Figure 2E, K, G and M).

In order to verify whether BYHWD promotes VEGFR2 activation, thereby inducing angiogenesis, SU5416 was administered to ICH animals in combination with BYHWD. We found that fewer pVEGFR2-positive microvessels and $\mathrm{BrdU}^{+}$nuclei in $\mathrm{vWF}^{+}$dilated vessels were present around the hemotoma of the BYHWD + SU5416treated group, compared with those of the BYHWD-treated group (Figure 2F, L, G and M).

Finally, we performed western blots to determine the effects of BYHWD on the phosphorylation of VEGFR2, and impact on the PI3K/Akt signaling pathway. The results showed that low levels of total VEGFR2 and pVEGFR2 were observed in the sham-operated brains (Figure 3N). Collagenase-induced ICH increased the activation ratio of VEGFR2, which was similar to that of the BYHWD group. This high ratio could be reversed by SU5416 $(P<0.05$, Figure $3 \mathrm{~N}$ and $\mathrm{O})$. Despite the similar activation ratio of VEGFR2 in both ICH and BYHWD administrated animals, the protein levels of total and activated VEGFR2 in the BYHWD group were about 2-fold higher than that of the ICH group $(P<0.05$, Figure $3 \mathrm{P})$.

\section{BYHWD promoted angiogenesis via pVEGFR2 mediated PI3k/Akt signaling pathway}

To further verify whether VEGFR2 activation is required for PI3K/Akt signaling transduction in BYHWD promoted angiogenesis, we examined the effect of SU5416 on the expression PI3K and the ratios of pAkt/Akt (Figure 3Q). The protein expression of PI3K and the ratios of pAkt/Akt were higher in the ICH group than that in SU5416-treated group $(P<0.05$, Figure $3 \mathrm{R}$ and $S)$. These positive effects were further increased by BYHWD treatment $(P<0.05$, Figure $3 \mathrm{R}$ and $\mathrm{S})$. However, the increased expression of PI3K and pAkt induced by BYHWD were markedly inhibited by SU5416 $(P<0.05$, Figure $3 R$ and $S)$.

\section{Discussion}

Our results demonstrate for the first time that blockade of VEGFR2 phosphorylation by SU5416 could also

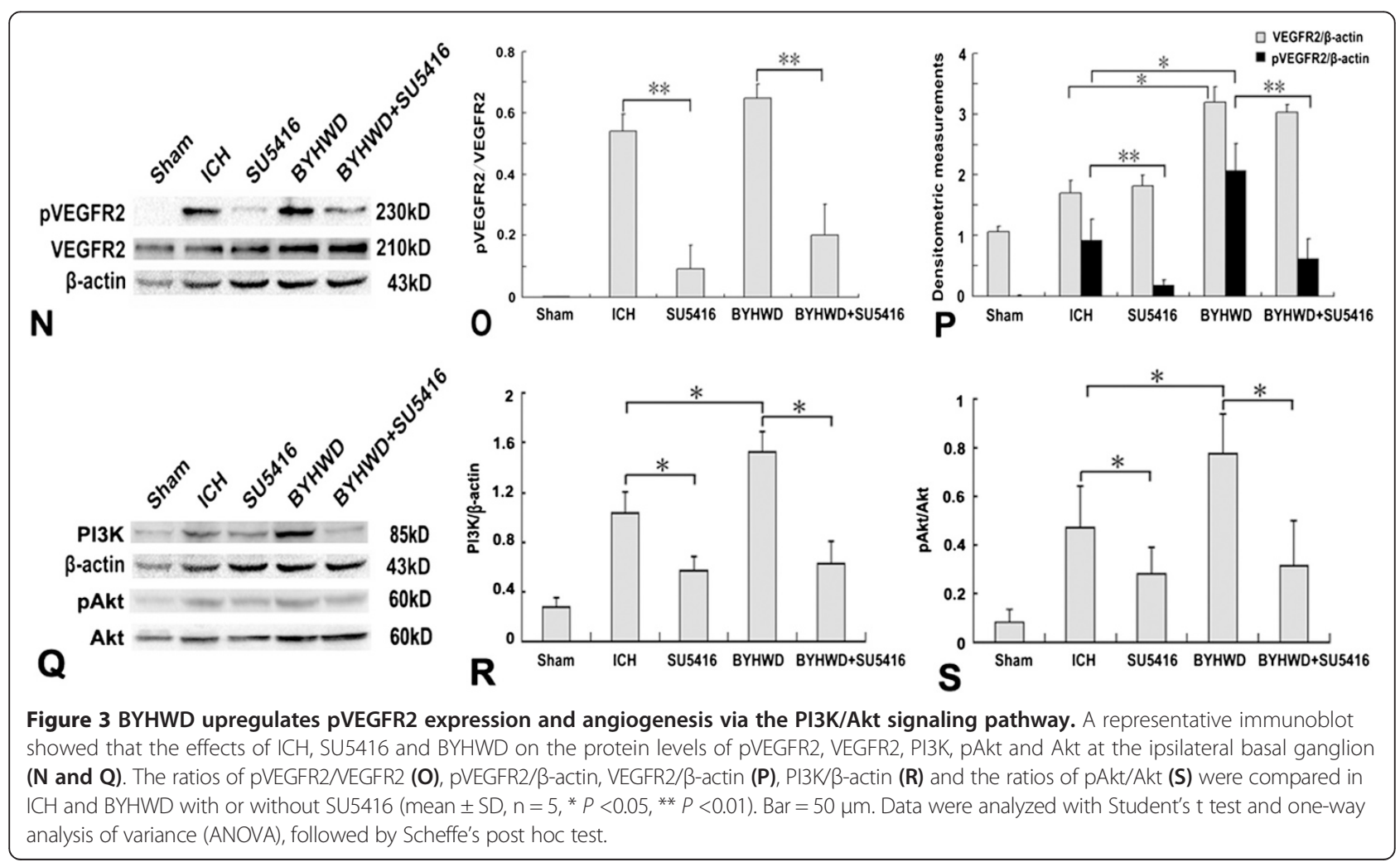


inhibit PI3k/Akt pathway, and decrease angiogenesis in $\mathrm{ICH}$ mouse brains and impede the amelioration of neurological function. Importantly, our results showed that BYHWD upregulated pVEGFR2 expression and increased the activation of the PI3K/Akt pathway, thus promoting angiogenesis and accelerating the recovery of neurological function. These effects were reversed by SU5416 treatment. These data supported that the underlying mechanisms of pro-angiogenic function of BYHWD might be involved with the enhancement of VEGFR2 phosphorylation through the PI3K/Akt pathway following hemorrhagic stroke.

VEGFR-2 is a type III transmembrane kinase receptor, which is mainly expressed in vascular and lymphatic endothelial cells. The critical role of VEGFR2 is demonstrated by the fact that VEGFR2 ${ }^{-1-}$ mice die at E8.5-9.5 due to defective development of yolk-sac blood islands, endothelial cells and hematopoietic cells [14]. Increased expression of VEGF and VEGFR2 in the basal ganglion lesion and proliferating ECs after $\mathrm{ICH}$ have been reported in our previous study, and their mRNAs are persistently elevated [13]. It is believed that VEGFR2 activation is a key step in regulating EC responses [29]. Suppression of VEGFR2 activity can enhance tumor regression and decrease vascular length and density as well as tumor vascularity. Additionally, inhibition of VEGFR2 signaling in cerebral ischemia and traumatic brain injury can aggravate brain injury by increasing cell death, and reducing EC proliferation [30-32]. Binding and activation of PI3K is required for phosphorylation of VEGFR2 at Tyr-801 and Tyr-1175 [33]. Thus, Akt appears to be critical for VEGF actions and a critical downstream target of PI3K. Activation of Akt was shown to promote angiogenic signals that can directly induce angiogenesis [34]. LY294002, a PI3K/Akt inhibitor, can also decrease EC proliferation and cell cycle progression [35].

To elucidate the potential role of VEGFR2 activation in angiogenesis in a mouse model of $\mathrm{ICH}$, we exploited the VEGFR2 inhibitor, SU5416 in our studies. This synthetic molecule contains an unsubstituted oxindole core and a dimethyl pyrrole attached to the indolin-2-one at the $\mathrm{C} 3$ position. Functioning as adenine mimetics, it acts as a competitive inhibitor at the catalytic domain of the tyrosine kinase, thus blocking the interaction between VEGF and VEGFR2 $[17,36]$. Immunohistochemistry revealed that the pVEGFR2 immunoreactivity in microvessels and BrdU-labeled nuclei in vessels adjacent to hematoma in the ICH group exceeded those in the SU5416-administrated group. Western blot also revealed that ICH increased the total and pVEGFR2 protein levels which were further evaluated by BYHWD treatment. Although BYHWD administration had a similar activation ratio of VEGFR2 to that of the $\mathrm{ICH}$, it increased almost two fold of total and activated VEGFR2 level over the
ICH. Moreover, VEGFR2 activation could be reversed by SU5416 in both ICH and BYHWD treatment groups. This agreed with a prior study on experimental brain contusions showing that the specific receptor inhibitor decreases capillary density via downregulation of pVEGFR2 [31]. Protein levels of downstream signaling molecules PI3K and pAkt showed a similar trend with pVEGFR2 across all of the five groups. This data suggested that activation of VEGFR2 was necessary for PI3K/Akt signaling transduction, and consistent with the report of Fournier et al. that SU5416 administration decreased the protein level of pAkt in the rat brain with VEGF microinfusion [37]. These results altogether suggested that upregulation of pVEGFR2 and coincident activation of PI3K/Akt pathway was partly involved in the $\mathrm{ICH}$-induced angiogenesis.

BYHWD, a traditional Chinese herbal prescription, has been used to treat paralysis and stroke in China for centuries [21]. We have previously demonstrated that VEGFR2 expression in brains of the BYHWD-treated group is higher than the ICH group, 7 days following stroke [24]. Radix Astragali is the main (monarch) drug in BYHWD constituting the largest concentration of 120 g. Astragaloside IV (AS-IV) is the major active constituent of Radix Astragali and has been shown to induce an angiogenic response in HUVECs by enhancing mRNA expression of VEGF and VEGFR2. This proliferation could also be suppressed by SU5416 specifically [38]. In the present study, we found more $\mathrm{BrdU}^{+}$nuclei in $\mathrm{vWF}^{+}$dilated vessels, and higher pVEGFR2 immunoreactivity in microvessels of BYHWD-treated animals than those in the $\mathrm{ICH}$ group. These results suggested that BYHWD was able to enhance angiogenesis and VEGFR2 activation. Moreover, SU5416 could reverse the effect of BYHWD on either the number of $\mathrm{BrdU}^{+} / \mathrm{vWF}^{+}$ vessels or the immunreactivity of p-VEGFR2. These results further suggested that BYHWD promoted angiogenic function by enhancing VEGFR2 phosphorylation following ICH.

VEGF and shear stress are two known factors that initiate the activation of VEGFR2 [39]. Our previous work showed that ICH-upregulated VEGF expression could be enhanced by BYHWD [13]. This effect has also been identified in ischemic animal models [22]. Therefore, VEGF increased by BYHWD may account for promoting VEGFR2 activation. Fluid shear stress is the other major factor that controls gene expression in vascular endothelia [40,41]. High flow (strong shear stress) drives VEGF expression to increase in parallel with its own receptor 2 , and their expression presents in a time- and dosedependent fashion [42]. Although CBF was reduced in the perihematomal zone and also remotely in the frontal cortex, this was only modest and transient. This decline, however, was resolved within $10 \mathrm{~min}$ and CBF restored to the normal level after ICH [43]. BYHWD was found 
effective in increasing capacity of microvascular reperfusion and CBF [44], which might be attributed to the increase of VEGFR2 activity.

As a sensitive and reliable indicator for detecting forelimb sensorimotor function, the mNSS tests were used to assess the neurological functions of mice, including motor ability, balancing, and alertness [45-47]. The corner turn and the food-fault test are easily performed, sensitive and objective tests for detecting impairments of sensorimotor function [45]. They have advantages on testing of multiple partial sensory and motor asymmetries associated with cortical or striatal dysfunction, including vibrissae sensory, postural and limb use biases documented in rat models $[48,49]$. The effects of BYHWD on neurological outcome improvement and infarction reduction in ischemic stroke have been reported [20]. In the collagenase model of hemorrhagic stroke in rats, the resulting hematoma triggers a series of events leading to secondary brain injury and severe neurological deficit [27]. As indicated by both clinical and preclinical studies, reduction of hematoma volume and decreased tissue injury improves outcomes in ICH models [50]. Other studies uncovered that angiogenic induction promotes neurogenesis, synaptogenesis, axonal sprouting and neuroblast migration to the lesion and further contributes to neurological recovery [51]. New vessel formation can improve blood supply to the injured region and regenerating neurons in the injured brain [52]. As demonstrated in our results, the improvement of neurological function in the ICH-treated and BYHWD-treated groups was coincident with the growth in the number of $\mathrm{BrdU}^{+} / \mathrm{vWF}^{+}$nuclei and pVEGFR2-immunoreactive microvessels. In contrast, fewer $\mathrm{BrdU}^{+} / \mathrm{vWF}^{+}$cells and pVEGFR2 labeled vessels could be observed in VEGFR inhibitor-treated counterparts, which showed a poor neurological outcome. This suggests that angiogenesis plays a central role in improving neurological function, and the pro-angiogenic effects of BYHWD may furthermore contribute to improving the phenotypes through boosting VEGFR2 activation.

\section{Conclusions}

In summary, our results demonstrate that the activation of VEGFR2 plays a pivotal role in $\mathrm{ICH}$-induced angiogenesis through the PI3K/Akt signaling pathway. Therefore, the pro-angiogenic mechanism of BYHWD in $\mathrm{ICH}$, may partly involve the upregulation of pVEGFR2 through a PI3K/Akt signaling mechanism.

\section{Competing interests}

The authors declare that they have no competing interests.

\section{Authors' contributions}

$\Pi T$ and HJC participated in the conception and design of the study. HJC, $A L Y$, and HJZ carried out the experiments. $C W, J K L$ and $Y L$ acquired of data. $\Pi \mathrm{T}$ and HJC analyzed and interpreted of data. HJC drafted the article. All authors read and approved the final manuscript.

\section{Acknowledgements}

This study was supported by grants from the National Natural Science Foundation of China (Grant Nos. 81473573, 81173175, 81202625), the Project for New Century Excellent Talent (NCET-11-0522), the Hunan Provincial Natural Science Foundation (Grant Nos. 14JJ2024), and Hunan TCM Project (Grant Nos. 201424)

\section{Author details}

${ }^{1}$ Institute of integrative medicine, Xiangya Hospital, Central South University, Changsha 410008, Hunan, China. ${ }^{2}$ Institute of neurology, Xiangya Hospital, Central South University, Changsha 410008, Hunan, China. ${ }^{3}$ Key Lab of Chinese Gan of SATCM, Changsha 410008, Hunan, China. ${ }^{4}$ Institute of Neurology, The First College of Clinical Medical Sciences, China Three Gorges University, Yichang 443003, Hubei, China.

Received: 11 February 2014 Accepted: 12 March 2015

Published online: 28 March 2015

\section{References}

1. Wang DZ, Talkad AV. Treatment of intracerebral hemorrhage: what should we do now? Curr Neurol Neurosci Rep. 2009;9(1):13-8.

2. Adeoye $\mathrm{O}$, Broderick JP. Advances in the management of intracerebral hemorrhage. Nat Rev Neurol. 2010;6(11):593-601.

3. Andaluz N, Zuccarello M. Recent trends in the treatment of spontaneous intracerebral hemorrhage: analysis of a nationwide inpatient database. J Neurosurg. 2009;110(3):403-10.

4. Wang J. Preclinical and clinical research on inflammation after intracerebral hemorrhage. Prog Neurobiol. 2010;92(4):463-77.

5. Wu H, Wu T, Li M, Wang J. Efficacy of the lipid-soluble iron chelator 2,2'-dipyridyl against hemorrhagic brain injury. Neurobiol Dis. 2012;45(1):388-94.

6. Chang CF, Cho S, Wang J. (-)-Epicatechin protects hemorrhagic brain via synergistic Nrf2 pathways. Ann Clin Transl Neurol. 2014;1(4):258-71.

7. Chopp M, Zhang ZG, Jiang Q. Neurogenesis, angiogenesis, and MRI indices of functional recovery from stroke. Stroke. 2007;38(2 Suppl):827-31.

8. Kojima T, Hirota Y, Ema M, Takahashi S, Miyoshi I, Okano H, et al. Subventricular zone-derived neural progenitor cells migrate along a blood vessel scaffold toward the post-stroke striatum. Stem Cells. 2010;28(3):545-54.

9. Tiwari A, Jung JJ, Inamdar SM, Choudhury A. Membrane trafficking and endothelial-cell dynamics during angiogenesis. In: Lawrite C, editor. Hematology - Science and Practice. Rijeka: Tech; 2012. p. 253-80.

10. Katoh M. Therapeutics targeting angiogenesis: genetics and epigenetics, extracellular miRNAs and signaling networks (Review). Int J Mol Med. 2013:32(4):763-7.

11. Shibuya M, Claesson-Welsh L. Signal transduction by VEGF receptors in regulation of angiogenesis and lymphangiogenesis. Exp Cell Res. 2006;312(5):549-60.

12. Zan $L$, Zhang $X, X i Y, W u H$, Song $Y$, Teng $G$, et al. Src regulates angiogenic factors and vascular permeability after focal cerebral ischemia-reperfusion. Neuroscience. 2014;262:118-28.

13. Tang T, Liu XJ, Zhang ZQ, Zhou HJ, Luo JK, Huang JF, et al. Cerebral angiogenesis after collagenase-induced intracerebral hemorrhage in rats. Brain Res. 2007;1175:134-42.

14. Roskoski Jr R. VEGF receptor protein-tyrosine kinases: structure and regulation. Biochem Biophys Res Commun. 2008;375(3):287-91.

15. Ferrara N, Gerber HP, LeCouter J. The biology of VEGF and its receptors. Nat Med. 2003;9(6):669-76.

16. Smith JK, Mamoon NM, Duhe RJ. Emerging roles of targeted small molecule protein-tyrosine kinase inhibitors in cancer therapy. Oncol Res. 2004;14(4-5):175-225.

17. Zhong XS, Zheng JZ, Reed E, Jiang BH. SU5416 inhibited VEGF and HIF-1alpha expression through the PI3K/AKT/p70S6K1 signaling pathway. Biochem Biophys Res Commun. 2004;324(2):471-80.

18. Kieran MW, Supko JG, Wallace D, Fruscio R, Poussaint TY, Phillips P, et al. Phase I study of SU5416, a small molecule inhibitor of the vascular endothelial growth factor receptor (VEGFR) in refractory pediatric central nervous system tumors. Pediatr Blood Cancer. 2009;52(2):169-76.

19. Liu H, Zhang GP, Song SJ, Ding MP, Zhou JF, Bie XD, et al. Clinical study on Zhuyu Tongfu serial recipe combined with acupuncture and massotherapy in treating hypertensive cerebral hemorrhage. Chin J Integr Med. 2005;11(3):167-72. 
20. Wei RL, Teng HJ, Yin $B, X u Y, D u Y, H e ~ F P$, et al. A systematic review and meta-analysis of buyang huanwu decoction in animal model of focal cerebral ischemia. Evid Based Complement Alternat Med. 2013;2013:138484.

21. Zhao LD, Wang JH, Jin GR, Zhao Y, Zhang HJ. Neuroprotective effect of Buyang Huanwu decoction against focal cerebral ischemia/reperfusion injury in rats-time window and mechanism. J Ethnopharmacol. 2012;140(2):339-44.

22. Cai G, Liu B, Liu W, Tan X, Rong J, Chen X, et al. Buyang Huanwu Decoction can improve recovery of neurological function, reduce infarction volume, stimulate neural proliferation and modulate VEGF and Flk1 expressions in transient focal cerebral ischaemic rat brains. J Ethnopharmacol. 2007;113(2):292-9

23. Hao CZ, Wu F, Shen J, Lu L, Fu DL, Liao WJ, et al. Clinical efficacy and safety of buyang huanwu decoction for acute ischemic stroke: a systematic review and meta-analysis of 19 randomized controlled trials. Evid Based Complement Alternat Med. 2012;2012:630124.

24. Zhang ZQ, Tang T, Luo JK, Huang JF, Yang QD, Li XQ, et al. Effect of qi-tonifying and stasis-eliminating therapy on expression of vascular endothelial growth factor and its receptors Flt-1, Flk-1 in the brain of intracerebral hemorrhagic rats. Chin J Integr Med. 2007;13(4):285-90.

25. Adjei AA. Novel small-molecule inhibitors of the vascular endothelial growth factor receptor. Clin Lung Cancer. 2007;8 Suppl 2:S74-8.

26. Narantuya D, Nagai A, Sheikh AM, Masuda J, Kobayashi S, Yamaguchi S, et al. Human microglia transplanted in rat focal ischemia brain induce neuroprotection and behavioral improvement. PLoS ONE. 2010;5(7):e11746.

27. Krafft PR, McBride DW, Lekic T, Rolland WB, Mansell CE, Ma Q, et al. Correlation between subacute sensorimotor deficits and brain edema in two mouse models of intracerebral hemorrhage. Behav Brain Res. 2014;264:151-60.

28. Cecatto RB, Maximino JR, Chadi G. Motor recovery and cortical plasticity after functional electrical stimulation in a rat model of focal stroke. Am J Phys Med Rehabil. 2014;93(9):791-800.

29. Lakshmikanthan S, Sobczak M, Chun C, Henschel A, Dargatz J, Ramchandran $\mathrm{R}$, et al. Rap1 promotes VEGFR2 activation and angiogenesis by a mechanism involving integrin alphavbeta(3). Blood. 2011;118(7):2015-26.

30. Shimotake J, Derugin N, Wendland M, Vexler ZS, Ferriero DM. Vascular endothelial growth factor receptor-2 inhibition promotes cell death and limits endothelial cell proliferation in a neonatal rodent model of stroke. Stroke. 2010;41(2):343-9.

31. Skold MK, Risling M, Holmin S. Inhibition of vascular endothelial growth factor receptor 2 activity in experimental brain contusions aggravates injury outcome and leads to early increased neuronal and glial degeneration. Eur J Neurosci. 2006;23(1):21-34.

32. Li WL, Fraser JL, YU SP, Zhu J, Jiang YJ, Wei L. The role of VEGFNEGFR2 signaling in peripheral stimulation-induced cerebral neurovascular regeneration after ischemic stroke in mice. Exp Brain Res. 2011;214(4):503-13.

33. Dellinger MT, Brekken RA. Phosphorylation of Akt and ERK1/2 is required for VEGF-ANEGFR2-induced proliferation and migration of lymphatic endothelium. PLOS ONE. 2011;6(12):e28947.

34. Jiang BH, Zheng JZ, Aoki M, Vogt PK. Phosphatidylinositol 3-kinase signaling mediates angiogenesis and expression of vascular endothelial growth factor in endothelial cells. Proc Natl Acad Sci U S A. 2000;97(4):1749-53.

35. Gharbi SI, Zvelebil MJ, Shuttleworth SJ, Hancox T, Saghir N, Timms JF, et al. Exploring the specificity of the PI3K family inhibitor LY294002. Biochem J. 2007:404(1):15-21.

36. O'Farrell AM, Yuen HA, Smolich B, Hannah AL, Louie SG, Hong W, et al. Effects of SU5416, a small molecule tyrosine kinase receptor inhibitor, on FLT3 expression and phosphorylation in patients with refractory acute myeloid leukemia. Leuk Res. 2004;28(7):679-89.

37. Fournier NM, Lee B, Banasr M, Elsayed M, Duman RS. Vascular endothelial growth factor regulates adult hippocampal cell proliferation through MEK ERK- and PI3K/Akt-dependent signaling. Neuropharmacology. 2012;63(4):642-52.

38. Zhang L, Liu Q, Lu L, Zhao X, Gao X, Wang Y. Astragaloside IV stimulates angiogenesis and increases hypoxia-inducible factor-1alpha accumulation via phosphatidylinositol 3-kinase/Akt pathway. J Pharmacol Exp Ther. 2011;338(2):485-91.

39. Urschel K, Garlichs CD, Daniel WG, Cicha I. VEGFR2 signalling contributes to increased endothelial susceptibility to TNF-alpha under chronic non-uniform shear stress. Atherosclerosis. 2011;219(2):499-509.

40. Urbich C, Stein M, Reisinger K, Kaufmann R, Dimmeler S, Gille J. Fluid shear stress-induced transcriptional activation of the vascular endothelial growth factor receptor-2 gene requires Sp1-dependent DNA binding. FEBS Lett. 2003;535(1-3):87-93.
41. van der Meer AD, Li Y, Duits MH, Poot AA, Feijen J, Vermes I. Shear stress induces a transient and VEGFR-2-dependent decrease in the motion of injected particles in endothelial cells. Biorheology. 2010;47(3-4):179-92.

42. Sho E, Komatsu M, Sho M, Nanjo H, Singh TM, Xu C, et al. High flow drives vascular endothelial cell proliferation during flow-induced arterial remodeling associated with the expression of vascular endothelial growth factor. Exp Mol Pathol. 2003;75(1):1-11.

43. Xi G, Keep RF, Hoff JT. Mechanisms of brain injury after intracerebral haemorrhage. Lancet Neurol. 2006;5(1):53-63.

44. Wu X, Li Y, Liu H, Yin Y, Zhao S, Guo Y. Microvessel changes in the gerbil hippocampus after cerebral ischemia and reperfusion by Buyang Huanwu decoction pretreatment. Neural Regen Res. 2011;6(9):656-60.

45. Reshef A, Shirvan A, Shohami E, Grimberg H, Levin G, Cohen A, et al. Targeting cell death in vivo in experimental traumatic brain injury by a novel molecular probe. J Neurotrauma. 2008;25(6):569-80.

46. Tsenter J, Beni-Adani $L$, Assaf $Y$, Alexandrovich AG, Trembovler $V$, Shohami $E$ Dynamic changes in the recovery after traumatic brain injury in mice: effect of injury severity on T2-weighted MRI abnormalities, and motor and cognitive functions. J Neurotrauma. 2008;25(4):324-33.

47. Zhang L, Schallert T, Zhang ZG, Jiang Q, Arniego P, Li Q, et al. A test for detecting long-term sensorimotor dysfunction in the mouse after focal cerebral ischemia. J Neurosci Methods. 2002;117(2):207-14.

48. Imbe H, Okamoto K, Donishi T, Senba E, Kimura A. Involvement of descending facilitation from the rostral ventromedial medulla in the enhancement of formalin-evoked nocifensive behavior following repeated forced swim stress. Brain Res. 2010;1329:103-12.

49. Kleim JA, Boychuk JA, Adkins DL. Rat models of upper extremity impairment in stroke. ILAR J. 2007:48(4):374-84.

50. Del Bigio MR, Yan HJ, Campbell TM, Peeling J. Effect of fucoidan treatment on collagenase-induced intracerebral hemorrhage in rats. Neurol Res. 1999;21(4):415-9.

51. Zhang ZG, Chopp M. Neurorestorative therapies for stroke: underlying mechanisms and translation to the clinic. Lancet Neurol. 2009;8(5):491-500.

52. Lee HJ, Kim KS, Park IH, Kim SU. Human neural stem cells over-expressing VEGF provide neuroprotection, angiogenesis and functional recovery in mouse stroke model. PLoS ONE. 2007;2(1):e156.

\section{Submit your next manuscript to BioMed Central and take full advantage of:}

- Convenient online submission

- Thorough peer review

- No space constraints or color figure charges

- Immediate publication on acceptance

- Inclusion in PubMed, CAS, Scopus and Google Scholar

- Research which is freely available for redistribution 